\title{
How and Why Choirs May Promote Health and Wellbeing?
}

\author{
Kari Batt-Rawden*
}

Associate Professor, Department of Health Sciences, Norwegian University of Science and Technology (NTNU), Gjøvik, Norway

${ }^{\star}$ Corresponding author: Kari Batt-Rawden, Associate Professor, Department of Health Sciences, Norwegian University of Science and Technology (NTNU), Gjøvik, Norway; Email: kari.batt-rawden@ntnu.no

Received: June 09, 2021; Accepted: June 17, 2021; Published: June 24, 2021

Recent research confirm that longevity and a healthy life is strongly influenced by belonging to closely knit communities or groups, that can give you a sense of meaning and of mastering in collective activities like nature and culture experiences. Increasingly more emphasis has been put on nature and cultural activities for maintaining health and quality of life [1-3], and may be linked to the building of social capital in local communities [4]. Health promotion is carried out by and with people, which improves both the ability of individuals to take action, and the capacity of groups, organizations or communities to influence the determinants of health (WHO, 1997). "Settings for health" represent the organizational base of the infrastructure required for health promotion. New health challenges mean that new and diverse networks need to be created to achieve intersectoral collaboration. Such networks should provide mutual assistance within and between countries and facilitate exchange of information on which strategies are effective in which settings. Public health research and practice should focus not only on factors causing disease and injuries (pathogenesis), but also on factors promoting health (salutogenesis) in the perspective of health promotion and prevention in different settings. Creative arts initiatives can be an effective way of meeting the growing calls for a shift of emphasis in mental health services, enhancing the significance of relationships and social support in the context of the well-being agenda. An adequate grasp of mutuality and social relationships is also important in addressing recent policy initiatives around loneliness [5]. Choral singing contributes to people changing their self-perception or maintaining their identity despite life affecting challenges or changes in living conditions [6]. Choral singing practice can be seen from a salutogenetic perspective that is, as something which promotes health and strengthens the healthy aspects of an individual in states of ease or dis-ease [7]. Singing can also be beneficial for those in the wider community who are affected by non-communicable diseases such as cancer [8]. Vitality was improved in those with a cancer diagnosis, and anxiety was reduced in cares and the bereaved. To use resources and capacities in communities by strengthening empowerment of the individuals that suffer from mental disorders and diseases, mostly anxiety and depression would also underline the importance of giving priority to the topic Public Mental Health Promotion in the light of new epigenetic research [3].

\section{Non-Pharmaceutical Interventions}

Quite often people would rather be prescribed non-pharmaceutical interventions than medication The Lancet Commission on Culture and Health states that as it is increasingly recognized that wellbeing has both biological and social elements, health care providers can only improve outcomes if they accept the need to understand the sociocultural conditions that enable people to be healthy and make themselves healthier - that is, to feel well [9], and then possibly recommend nonpharmaceutical interventions. Seven years of research by the James Lind Alliance into the clinical research priorities of patients, carers, and clinicians indicated that $72 \%(103 / 126)$ of treatment priorities were non-pharmacological and that often people would rather be prescribed non-pharmaceutical interventions than medication [10]. Marmot has described social exclusion as "deprivation on stilts" [11] to accentuate how damaging it is for the individual and society. He advocates for any changes that could help tackle social exclusion, and it could be argued how choirs would be 2 fertile grounds for further research for a potential role in health promotion by facilitating a pathway to social inclusion. In response to this, 'social prescribing' is becoming more prevalent, whereby people presenting to primary care are linked with various sources of support within the local community, from gardening projects to table tennis clubs, and choirs can act as another potential non-pharmacological 'tool' [12]. Leisure time is increasingly important for emotional wellbeing, informal learning and identity formation among children and youth. Contemporary societies are characterized by increasing individualization, affecting identity formation, wellbeing and sense of coherence and belonging. The institutionalization of childhood and education/knowledge has increasingly compartmentalized children and young people into exclusive spheres set apart from the adult world, placing them in an age-segregated social order, at the cost of being included in an intergenerational social order [13]. Health benefits from musicking ${ }^{1}$ [14] may reduce stress, anxiety, depression by building coping capabilities, resilience social inclusion and renewed strength [15].

Musicking: To music is to take part, in any capacity, in a musical performance, whether by performing, by listening, by rehearsing or practicing, by providing material for performance (what is called composing) or by dancing. (Small, 1998:9). See also David Elliott's definition of Musicing: all human action related to [music.]" [14]. 


\section{Future Studies}

Despite widespread anecdotal evidence that singing has a positive effect on health and wellbeing, and the burgeoning number of studies suggesting potential benefits in many diverse fields, recent systematic reviews have identified that the quality of evidence is sometimes poor. McNamara's Cochrane review of the singing and COPD literature suggested that the quality of evidence is low to very low. This was thought to be due to the small size and the low number of randomized controlled trials [16]. Other methodological limitations have meant that outcome measures vary or there are no consistent changes in outcome measures. Randomised controlled trials of singing interventions suffered from attrition as people who wanted to sing were not allocated it, or the singing 'intervention' offered was too short, too finite, or simply not appealing. Clark and Harding's [17] systematic review of the psychosocial outcomes of singing interventions concluded that more qualitative studies were needed. The results of the six studies that have been carried out since then are interesting, convergent, but (naturally) inconclusive. In order to explore such ecological functions of choral singing, participant observation is a good strategy in addition to the ethnographic interview. Through participant observation of the choral singing practice and events, we can investigate how choral singing is imbricated into their social networks and how it expands their social world.

\section{References}

1. Hansen E, Sund E, Krokstad S (2015) Cultural activity participation and associations with self-perceived health, life-satisfaction and mental health: the Young HUNT Study, Norway. BMC Public Health 15.

2. Cuypers K, Krokstad S, Holmen TL, Margunn SK, Lars OB, et al. (2013) Patterns of receptive and creative cultural activities and their association with perceived health, anxiety, depression and satisfaction with life among adults: the HUNT study, Norway. J Epidemiol Community Health 66 : 698-703. [crossref]

3. Tellnes G, Batt-Rawden KB, Christie WH (2018). Nature Culture Health Promotion as Community building. Journal "Herald of the International Academy of Science. \#1. Russian Section" (HIAS.RS). Herald of the International Academy of Science. Russian Section". vol. 1 (1).
4. Campbell C, Gillies P (2001) Conceptualizing 'social capital' for health promotion in small local communities: a micro-qualitative study. Journal of Community \& Applied Social Psychology. 11 : 329-346.

5. Sturgeon S (2006) Promoting mental health as an essential aspect of health promotion. Health Promotion International 21 : 36-41. [crossref]

6. Balsnes AH (2010) Choir research - a Norwegian perspective. In : Geisler U, Johansson K (eds.), Choir in Focus. Pg : 16 - 19, Bo Ejeby Publisher.

7. Clift S, Jennifer N, Raisbeck M, Whitmore C, Morrison I (2010) Group singing, wellbeing and health: A systematic mapping of research evidence. UNESCO Observatory, Faculty of Architecture, Building and Planning, The Melbourne Refereed E-journal 2

8. Batt-Rawden KB, Andersen S (2018) "Singing has empowered, enchanted and enthralled me" Choirs for Wellbeing?". Health Promotion International 3.

9. Napier D, et al (2011) Culture and Health Lancet Commission. The Lancet.

10. Crowe S, Fenton M, Hall M, Cowan K, Chalmers I (2015) Patients', clinicians' and the research communities' priorities for treatment research: there is an important mismatch. Research Involvement and Engagement 1.

11. Marmot M (2015) The Health Gap: the Challenge of an Unequal World. Bloomsbury, London, UK.

12. Ruud E (2013) Can music serve as a "cultural immunogen"? An explorative study. International journal of qualitative studies on health and well-being. $8: 205-209$. [crossref]

13. Beynon C, Lang J (2018) The More We Get Together, The More We Learn: Focus on Intergenerational and Collaborative Learning Through Singing. Journal of Intergenerational Relationships $16: 45-63$.

14. Small C (1998) Musicking. The Meanings of Performing and Listening. Weslyan Press. London.

15. Batt-Rawden KB (2018) The fellowship of Health Musicking: A Model to Promote Health and Well-Being. Music and Public Health - A Nordic Perspective. Bonde LO, Theorell T (eds) Springer Verlag.

16. Williams E, Dingle G, Clift S (2018) A systematic review of mental health and wellbeing outcomes of group singing for adults with a mental health condition. European Journal of Public Health 28 : 1035-1042. [crossref]

17. Clark I, Harding K (2012) Psychosocial outcomes of active singing interventions for therapeutic purposes: A systematic review of the literature. Nordic Journal of Music Therapy $21: 80-98$ 